\title{
A 3D Classical Object Viewer for Device Compatible Display
}

\author{
Muhammad Sajid Khan, Tayyab Rehman, Andrew Ware, Muhammad Jehanzeb, Muhammad Imran \\ Babar, and Muhammad Abubakar Yamin
}

\begin{abstract}
The paper presents a 3D Android-based classical object viewer system that improves user experience when viewing 3D datasets. The system is well suited to 3D simulated images and facilitates a more accurate list of images in response to search-based quarries using Android's SearchView widget. Existing 2D object viewer systems are unable to display the simulated images accurately and older 3D object viewing systems face performance-related challenges. The developed Android system reduces the issues of performance and improves the flexibility in viewing rotation for 3D environments. It also provides better user experience and versatility to 3D object viewers through different paths and channels. The effectiveness of the developed system has been demonstrated using standard datasets.
\end{abstract}

Index Terms-3D interaction, 3D viewer, user experience, usability.

\section{INTRODUCTION}

3D display technology has developed rapidly in the last decade and classical 3D object viewers are important in many emerging applications. The technology incorporates many useful features that facilitate viewing of various image types (including those constructed from polygon shapes and those that are simulated) and enable analytic capabilities while providing intuitive use.

In computer graphic applications, a mesh is a combination of vertices, faces and edges that describes the various shapes and objects in a scene. When the surface is planar, surface mesh models are not sufficient to facilitate an accurate reconstruction of $3 \mathrm{D}$ objects without effecting resolution and quality. Moreover, in general, several problems arise with 3D data representation, that includes rendering, comparison, storage and retrieval, because of poor content and structure of 3D datasets [1]. For the purpose of reconstruction, the concept of points and lines [2] with meshes is not entirely suitable for designing of 3D models and views. A method of 3D reconstruction that consists of constructive solid geometric (CSG) shapes, which deal with the surface, is more flexible and faster to process than lines and patch based methods when constructing simple primitives and geometric

Manuscript received March 6, 2020; revised May 28, 2020.

Muhammad Sajid Khan, Muhammad Jehanzeb, and Muhammad Imran Babar are with Army Public College of Management \& Sciences, Rawalpindi, Pakistan (e-mail: imran.babar77@gmail.com).

Tayyab Rehman is with Institute of Information Technology, PMAS Arid Agriculture University, Rawalpindi, Pakistan (e-mail: rehmantayyab786@gmail.com).

Andrew Ware is with the University of South Wales, United Kingdom (e-mail: andrew.ware@southwales.ac.uk).

Muhammad Abubakar Yamin is with Istituto Italiano di Technologia, Genova, Italy (e-mail: abubakar.yamin@gmail.com). shapes.

Many online web-based systems are available for 3D viewers, but most of them depend on backend servers to process the images, which requires time and internet connectivity to upload the images and receive back the results. These systems do not provide an offline (no internet connectivity) processing facility they also lack important features such as zoom in and zoom out. Moreover, they often have difficulties in achieving high-level performance and the quality when viewing objects can be poor.

The developed system provides a better experience when viewing a $3 \mathrm{D}$ model in a range of different scenarios, enabling users to control the interface of the architecture. The system also supports a 3D modelling approach, allows for the import of standard 3D datasets, and facilitates the adjustment of the textural intensity and placement of light source within the skybox tool kit. It also provides flexibility [3] to users by giving them the ability to add more features to the desired output to meet the requirements of $3 \mathrm{D}$ scenes. Once the users have setup the expected scene, the application requires the camera controller to move around the structure amidst the scene and presents the structure from all possible views.

The developed 3D classical object viewer provides a quick way of previewing models without having to wait for the main 3D application to load [4], [5]. Due to these flexibilities, installation of specialist software is not required. The system also offers the usual choice of textured and wireframe display modes, and the option of displaying the image background grid or the model's bounding box. The display view empowers the users to look at the datasets from different perspectives displayed on a screen in the viewport, where users can also control the display characteristic, such as lighting and image intensity. The system also has additional features for displaying still images on a screen. While the user cannot configure or change the design of the dataset via the viewport, the scale of the image can be altered. The user is also able to either start the $3 \mathrm{D}$ viewer automatically on page load or on-demand by explicitly clicking on the 3D viewer to initialise [6].

In older systems, viewers will show an image preview of a dataset, inviting the user to interact with the system in order to facilitate the 3D capabilities. This requires the installation of 3D model architecture. When utilizing glTF files, some 3D models can be very large, so an offloading methodology is often used to remove application related constraints from the process. The developed 3D system enables the datasets and pictures to be rotated through 360-degrees, providing improved rotational capability, flexibility and speed. This provides the users with smart interaction through intuitive understanding of the interface. The system allows for the 
manipulation of the 3D objects using an interactive approach. The system responds to user touch events, using the gesture mode in smart phones to deal with 3D models and objects [7] Moreover, various operations on the datasets, including zooming and rotation in multiple directions and views, are facilitated. The need for input devices such as keyboards, digital pens, mice, and joysticks, when working with geometric shapes and models, is also eliminated.

In the system implementation, Wikitude SDK for Android [8] was used to convert images into binary form, making them suitable for display and manipulation. The resultant file is optimized for fast rendering of models and objects to the screen. The Human finger step algorithm [9] is used to adjust the rotation and capture user gesture, hence enhancing usability. A 3D environment is controlled by the the outer boundary description of the object using a surface detection algorithm [10].

\section{RELATED WORK}

The $3 \mathrm{D}$ reconstruction and $3 \mathrm{D}$ viewers are the core topic in computer image technology with increasing the demand for visualization experiences of the users in different devices like camcorders, Smartphone and digital displays. The 3D viewer's based systems are valuable in the study of human anatomy, care simulation, communication and trainer education. It becomes more useful because of cloud storage and multimedia technologies with high popularity in the social media for image, video, augmented reality, viewpoint and virtual reality. The values of images and video processing have been rapidly growing but all of them, not the good quality which is affected at several stages for quality like transmission, composition and destruction. The qualities of images have very important for user viewing and experiencing with system. Numerous kinds of images have quality assessment metrics provided by the researchers to measure the quality, restoration and enhancement algorithm of an image. The quality of $2 \mathrm{D}$ and $3 \mathrm{D}$ images are very important for the advanced system and an issue to maintain the quality for viewing and experiencing. The fidelity is the main factor that affects the 2D images while stereoscopic weak the visual comfort of the 3D images that become increases the structure views problem. It is found from the previous studies that the distortion affects the face recognition and face detection process of the system, and for purposes number of reconstructions, systems are introduced to restore and improved the images. The new user $3 \mathrm{D}$ viewers experience is trend come out with $2 \mathrm{D}$ to $3 \mathrm{D}$ reconstruction methods that have highly applied in advanced 3D based system.

The 3D models or motion images are the collection of 2D images or frames that transferring 2D motion into 3D motion. Many methods are derived for the conversion of 2D images into 3D images for various purposes in different applications. Where some algorithms have focuses on the time and performance with improvement in the models for views and experience. Generally, two main categories are used for the conversion into 3D images. The first category based on the process with multiple images through camera and another category is the direct methods into 3D. These methods provide the productive result with special equipment and system which increase the cost of the system. In last year's, $3 \mathrm{D}$ reconstruction becomes more appropriate with using of laser scanning technology and it focuses on speed, resolution and accuracy. It mostly reconstructs the $3 \mathrm{D}$ objects, scene, and large size of buildings with interior and exterior, archaeology site and historical building. The laser scanning based reconstruction undergoes through Image acquisition, Registration, Data processing which consists of elimination and construction. The initial registration includes wide range of scan methods with geometric shapes for extraction of features and matching to allow the $3 \mathrm{D}$ reconstruction, where a number of descriptors use for registration in $3 \mathrm{D}$ and recognition in many applications. The surface representation is useful for the laser scanning based application. The other important category is fine registration that transforms the good estimation for scan points and optimally aligns the point sets. The ICP based algorithms are productive for fine registration and of 3D data sets. It improves the performance when any pose or side view of an image which expected to be guess or recognized. The multi stereo images concept is the emerging in $2 \mathrm{D}$ to $3 \mathrm{D}$ reconstruction technology that's are more efficient and using the concept of dense for reconstruction where views the objects from multiple views. It uses a large number of images for different techniques to produce 3D models. The visual hull reconstruction is possible with silhouette methods based on the multi-camera environment for reducing the computation time and efficiency. Another sub-type is the space curve reconstruction that stereo images into account and recover the $3 \mathrm{D}$ images with photometric stereo techniques.

The conversion of 2D to 3D data is complex in processing and visualization. So it facilitate a new area with digital viewer that explore the presentation and useful in many applications. The Ryan provide the idea that birth to the development and foundation to the interactive system its support multiple 3D interactive system based on the straight graphic. It collaborate the mixed reality, visual interaction with lacked well designed.

There are only a limited number of systems available that handle Touch events based on 2D input of objects. Similarly, for 3D systems, there are a limited number of web-based systems. In this paper, the foundation is laid for an application system that can handle the input of 3D models and objects.

In this regard, Michael M. Kazhdan et al. [11] presented a concentration-based approach. However, it is based on the items and list of control features such as the 3D models and objects that requires a point-based approach that reduces the incremental development in the construction of objects. When displaying detailed 3D models and objects in a coordinate system a sketch profile consisting of limited information is used [12]. This not only improves the visual performance but also the structuring and model display of the objects.

The interactive features of this application, such as rotation and zooming functions, are not easy to perform. Hence, a transformation process of construction of $3 \mathrm{D}$ models and pictures is used by the control functions to utilize the productively of the application without using the standard gadgets of a PC. Hence, it is a versatile approach with little latency and operates by using finger touch functions. 
The new system provides an environment in which a model-based methodology utilizes the object's 3D structure alongside its rendering. Using the geometrical shape of the $3 \mathrm{D}$ objects, control touch events can alter their position and orientation [13]. The model methodology utilizes edge identification for development of 3D models. To facilitate flexibility, it was important that the new system had the capability to support touch events in different direction. Thus during object viewing, a fixed-measure search window is set relating to the client's hand estimation with the goal that it incorporates some portion of hand on the screen ratio feature [14].

\section{Methodology}

This section describes the 3D image viewer developed to display various 3D content, including models and objects, using touch event functionality in an offline mode. The image viewer enables the effective use of a device's viewport (the viewport is the user visible area of a screen and is device dependent) something that is currently lacking in state-of-the-art Android systems. The Wikitude API is used to import a model or objects using the fingertip algorithm to provide efficient and precise control.

The system overcomes some of the shortfalls of traditional input mediums that are not able to deliver the desired results on mobile phones. This system allows the user to determine various properties of the displayed image such as the level of zoom, as well as the parameters of its operation. Dealing with 3D models or objects is a very difficult task for input gadgets, as they need to perform several operations. Similarly, they require several human-machine interactions involving elaboration and translations. Fig. 1 illustrates the system's output.

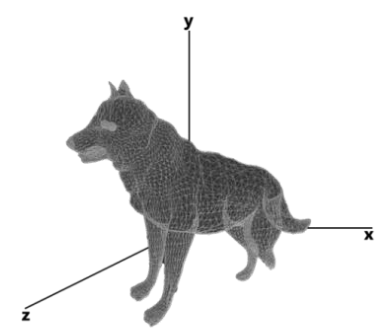

Fig. 1. Output of Android app as a single image for the object.

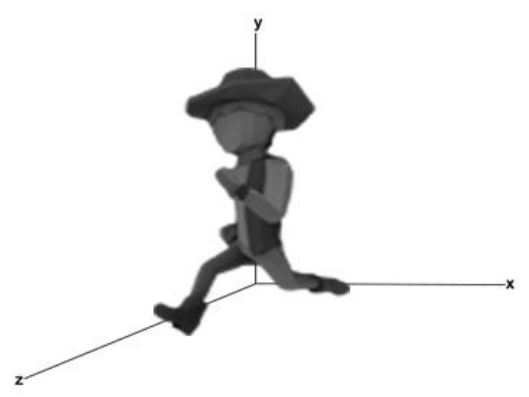

Fig. 2. Output of Android app as a single image for the object.

\section{A. Three Dimensional Point and Detection Parameters}

This developed system provides better accuracy when viewing $3 \mathrm{D}$ models. However, in terms of the processing and rotation-based properties of an object, it diverts focus of attention from the screen to the model. In order to overcome this problem, the location of the object or model remains constant on the screen. For 3D rotation, a 3D surface detection algorithm [15] is often used.

The main highlights of the featured algorithm include 3D filtering (used to analyze objects and environments in different applications), dependability (location-aware focus for smooth view recreation), and neighbor-awareness (in 3D data sets, neighbor and its characteristics can be found using neighborhood techniques e.g. spherical neighborhoods, cylindrical neighborhoods).

\section{B. $3 D$ Object Viewer}

The system makes use of Adobe XD to provide a professional user interface that enables its control by the user.

\section{Finger Tracking}

The purpose of the touch detection algorithm [16] is to overcome the serious problem of large model and animation shadow effects. After gathering the data for the models, classifying the touch events makes the system more flexible and intuitive to use. The developed system detects a touch event on the screen while rotating the $3 \mathrm{D}$ objects. This enables the user to perform their desired actions results on it.

\section{EXPERIMENTAL RESULTS}

The developed system was trialed on various datasets to provide real-time input in different positions; producing accurate results across a wide range of inputs and outputs from applications with varying requirements. The graphs shown in Fig. 3 demonstrate various results obtained where the $y$-axis shows the measurement flow in accuracy, errors and performance.

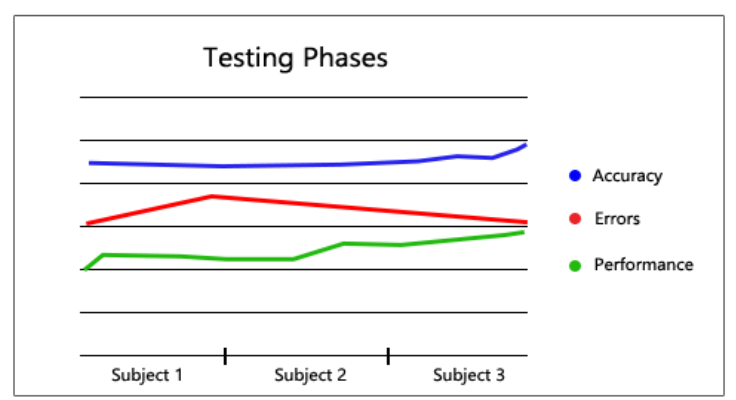

Fig. 3. Graphical representation of the experimental results.

The system's tracking and positioning of 3D models using the system is illustrated in Fig. 4 and Fig. 5, where the objects are rotated in $3 \mathrm{D}$ for rotation and zooming purposes. The results in Table I show the accuracy achieved.

TABLE I: PERFORMANCE AND ACCURACY OF THE SYSTEM

\begin{tabular}{|c|c|c|}
\hline \multicolumn{3}{|c|}{ Performance and Accuracy of Developed Method } \\
\hline & Minimum accuracy & Maximum accuracy \\
\hline Loading Object & $60 \%$ & $100 \%$ \\
\hline Zooming & $70 \%$ & $96.6 \%$ \\
\hline Panning & $70 \%$ & $100 \%$ \\
\hline Rotating & $80 \%$ & $98 \%$ \\
\hline
\end{tabular}

3D data rendering involves the display of data and models. In $3 \mathrm{D}$ software-based analysis, the focus is to improve the 
performance of the 3D viewer system. Many packages incorporating such software are used to display and convert 3D data files. When appraising the display models, one possible approach is to categorize the attributes and design metrics of the $3 \mathrm{D}$ datasets. In respect to the developed system, the salient attributes are its flexibility and adaptability. While testing proved the versatility of the new system, nonetheless the user interactions of rotation and scaling were difficult to perform when using 3D coordinate systems.



Fig. 4. Illustrative output from a system.



Fig. 5. Output of a system.

When trialed using the same dataset, the newly developed system demonstrated improvements in comparison to existing systems. These existing systems [17] work well when dealing with rotation and direction related changes. However, they are web-based and do not offer offline usability.

While several of these web-based methods incorporate advanced features such as panning and zooming, they often suffer degradation in loading times due to latency in internet connectivity. To ameliorate these issues, the developed application makes use of Wikitude SDK. Moreover, it includes touch screen event tracking to facilitate functionality such rotation, 360 rotations, zooming and panning. In addition, the touch event technology replaces traditional peripherals such as mouse and keyboard. The system is accurate and compatible with existing displays thus suitable for a wide range of applications.

\section{CONCLUSION}

3D modeling is getting more importance in advanced systems and applications nowadays due to development and innovation in $3 \mathrm{D}$ visualization. It enables the less cost and provide more flexibility in design and structure. It is the platform to display the different sides and direction of object. It's more realistic, simple and easily interacted for the end users. 3D modeling is highly applicable in virtual actors, augmented reality and virtual reality. It's reduces the complexity of architecture and improve the interior design. So that $3 \mathrm{D}$ objects and 3D models are becoming increasingly popular in different areas of modeling. There is lot of online applications for 3D viewers which consist of connectivity issues, visualization problem and performance that affects due to large size of datasets. However, currently no built-in apps allow for the import, manipulation, and display of 3D objects on a mobile phone. Moreover, traditional tools and input devices such as mouse and keyboard do not meet the requirements for dexterous interaction with the device. It has the problem of compatibility with different devices.

The paper describes a 3D Android based viewing system that incorporates a simple finger control interface through which the user can perform a range of operations with ease and comfort. It works in off line and provides good performance, maintain the usability and keep better user experience.

\section{CONFLICT OF INTEREST}

The authors declare no conflict of interest.

\section{AUTHOR CONTRIBUTIONS}

Muhammad Sajid Khan conceived the research idea articulated in the paper and has coordinated the research process. Tayyab Rehman helped develop the framework used to obtain the experimental results. Andrew Ware provided ideas for progressing the work and helped in the writing of the paper. Muhammad Jehanzeb and Muhammad Imran Babar aided in verifying the veracity of the results. Muhammad Abubakar Yamin provided an analysis of the pertinent literature.

\section{ACKNOWLEDGMENT}

The authors are grateful to their home institutions for the support given during the collaborative research reported in the paper.

\section{REFERENCE}

[1] M. Kenton and P. Bajcsy, "An overview of 3d data content, file formats and viewers," National Center for Supercomputing Applications, vol. 1205, p. 22, 2008.

[2] J. Liu, C. Yu, and X. Tang, "Decomposition of complex line drawings with hidden lines for 3D planar-faced manifold object reconstruction," IEEE Transactions on Pattern Analysis and Machine Intelligence, vol. 33, no. 1, pp. 3-15, 2010.

[3] G. Lv, B. Zhao, Z. Liu, H. Deng, and F. Wu, "A 2D/3D compatible display based on polarization modulator," Optik, vol. 184, pp. 485 489, 2019.

[4] M. S. Khan, M. Rabbiya, S. Ayesha, and N. Adnan, "A new 3D eyeball tracking system to enhance the usability of page scrolling," Optik, vol. 185, pp. 1270-1276, 2019.

[5] G. Fabrizio, M. Callieri, M. Dellepiane, M. Charno, J. Richards, and R. Scopigno, "Web-based visualization for 3D data in archaeology: The ADS 3D viewer," Journal of Archaeological Science: Reports, vol. 9, pp. 1-11, 2016.

[6] Y.-T. Lee and O. Taisuke, "OpenMX viewer: A web-based crystalline and molecular graphical user interface program," Journal of Molecular Graphics and Modelling, vol. 89, pp. 192-198.

[7] B. Matteo, M. Tarini, N. Pietroni, M. Livesu, and P. Cignoni, "HexaLab. net: An online viewer for hexahedral meshes," ComputerAided Design, vol. 110, pp. 24-36, 2019. 
[8] V. D. Heijden, G. W. Theodorus, P. S. Newton, C. Benien, and F. Gremse, "Transferring of $3 \mathrm{~d}$ viewer metadata," U.S. Patent Application 13/201,809, December 8, 2011.

[9] G.-J. Lv, B.-C. Zhao, Z.-S. Liu, H. Deng, and F. Wu, "A 2D/3D compatible display based on polarization modulator," Optik, vol. 184, pp. $485-489,2019$.

[10] X. Yin and X. Ming, "Finger identification and hand posture recognition for human-robot interaction," Image and Vision Computing, vol. 25, no. 8, pp. 1291-1300, 2007.

[11] K. W. Allison, W. Li, M. M. Kazhdan, W. T. Corrêa, A. Finkelstein, and T. A. Funkhouser, "Non-photorealistic virtual environments," in Proc. the 27th Annual Conference on Computer Graphics and Interactive Techniques, 2000, pp. 527-534.

[12] K.-H. Chang, "Chapter 3-solid modeling, e-design," Computer-Aided Engineering Design, pp. 125-167, 2015.

[13] B. Wu, R. L. Klatzky, and G. Stetten, "Visualizing 3D objects from 2D cross sectional images displayed in-situ versus ex-situ," Journal of Experimental Psychology: Applied, vol. 16, no. 1, p. 45, 2010.

[14] G. Fabrizio, M. Callieri, M. Dellepiane, M. Charno, J. Richards, an R. Scopigno, "Web-based visualization for 3D data in archaeology: The ADS 3D viewer," Journal of Archaeological Science: Reports, vol. 9, pp. 1-11, 2016.

[15] F. Goodarzi and F. Z. B. Rokhani, "Analysis of the effect of ethnicity, color and gender on multi view face emotion recognition," Journal of Software Engineering \& Intelligent Systems, vol. 4, issue 1, April 2019.

[16] M. M. Kazhdan, "Shape representations and algorithms for 3D model retrieval," Ph.D diss., Princeton University, 2004.

[17] S. S. Rautaray and A. Anupam, "Vision based hand gesture recognition for human computer interaction: A survey," Artificial Intelligence Review, vol. 43, no. 1, pp. 1-54, 2015.

Copyright $(92020$ by the authors. This is an open access article distributed under the Creative Commons Attribution License which permits unrestricted use, distribution, and reproduction in any medium, provided the original work is properly cited (CC BY 4.0).

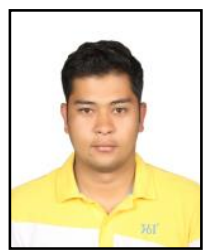

Muhammad Sajid Khan is an assistant professor of software engineering at the Army Public College of Management \& Sciences, Rawalpindi, Pakistan. His research interests include human computer interaction (HCI), detection, recognition and identification.

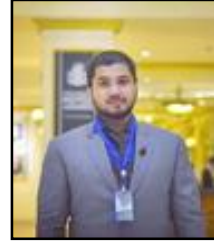

Tayyab Rehman is a BS student of computer science at University Institute of Information Technology PMAS Arid Agriculture University, Rawalpindi, Pakistan. His research interest includes web development and software designing.

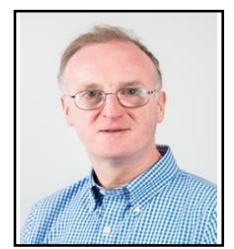

Andrew Ware is a professor of computer science at the University of South Wales in the United Kingdom. He is the Editor in Chief of the Annals of Emerging Technologies in Computing (AETiC). His research centres on the application of AI to help solve real world problems.

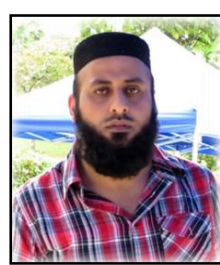

Muhammad Jehanzeb is an assistant professor of computer science at the Army Public College of Management \& Sciences, Rawalpindi, Pakistan. His research area includes writing recognition, documents analysis and biometrics.

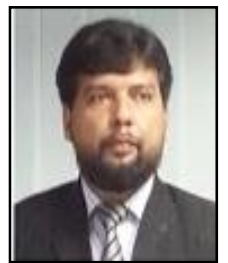

Muhammad Imran Babar is an assistant professor of software engineering at the Army Public College of Management \& Sciences, Rawalpindi, Pakistan. He is the Editor in Chief of Journal of Software Engineering and Intelligent Systems (JSEIS). His research interests include Software engineering, software requirements engineering, empirical software engineering, software testing, expert systems, and artificial intelligence.

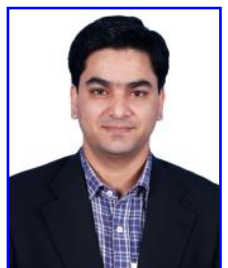

Muhammad Abubakar Yamin is a $\mathrm{PhD}$ student with the Pattern Analysis and Computer Vision Research Group at the Istituto Italiano di Technologia, Genova, Italy. He worked as a senior lecturer at Department of Computer Engineering, Bahria University from Aug 2013 to Oct. 